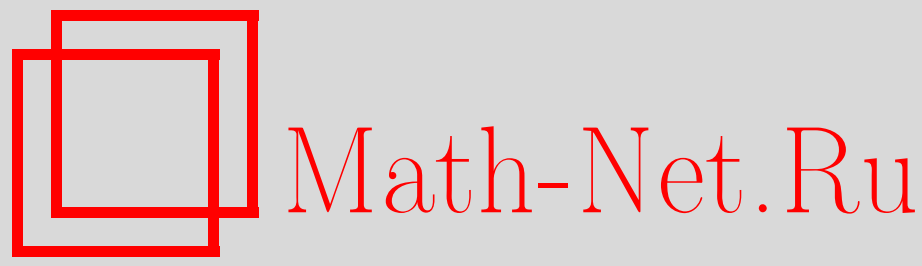

Э. А. Аринштейн, Оператор Мацубары модели Фрелиха, TMФ, 2009, том 161, номер 2, 243-255

DOI: https://doi.org/10.4213/tmf6435

Использование Общероссийского математического портала Math-Net.Ru подразумевает, что вы прочитали и согласны с пользовательским соглашением http://www . mathnet.ru/rus/agreement

Параметры загрузки:

IP : 3.85 .7 .115

26 апреля 2023 г., $17: 19: 15$

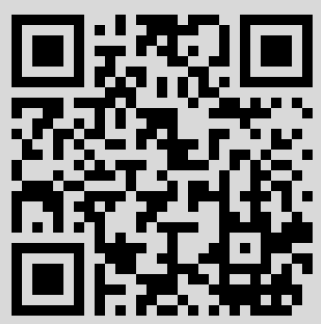




\section{ОПЕРАТОР МАЦУБАРЫ МОДЕЛИ ФРЕЛИХА}

Рассмотрена теория возмущений для оператора Мацубары модели Фрелиха системы электронов и фононов с использованием метода производящего функционала. Получены выражения, определяющие возмущенный гамильтониан электрон-фононной системы и эффективный гамильтониан электронной системы при усреднении по фононным состояниям.

Ключевые слова: оператор Мацубары, модель Фрелиха, матрица плотности, производящий функционал, теория возмущений.

\section{1. ВВЕДЕНИЕ}

Даже в системе невзаимодействующих частиц квантовые эффекты могут играть весьма существенную роль и приводить к таким свойствам термодинамических функций, которые принципиально отличаются от свойств классических систем. Квантовые эффекты в системе взаимодействующих частиц также могут оказаться весьма существенными, однако их исследование является более сложной задачей.

При статистическом описании квантовой системы взаимодействующих частиц чаще всего в современной физике используется весьма продуктивная идея квазичастиц, взаимодействие между которыми можно рассматривать как малое возмущение [1]. Параметры квазичастиц и эффективного взаимодействия между ними вводятся в теорию на основе анализа экспериментальных данных или на основании общих соображений. Однако такой подход не является универсальным, он может оказаться неадекватным в случае интенсивного взаимодействия между квазичастицами, когда само понятие квазичастиц оказывается определенным недостаточно четко. Из этого обстоятельства вытекает актуальность расчета статистических и термодинамических свойств квантовой системы взаимодействующих частиц без использования идеи квазичастиц.

Прямой расчет статистической суммы и термодинамических свойств квантовой системы взаимодействующих частиц требует преодоления весьма серьезных технических проблем. Обычно при этом используется термодинамическая теория возмущений, основанная на различных методах разложения оператора Мацубары. Для их

${ }^{*}$ Тюменский государственный университет, Тюмень, Россия. E-mail: earin@inbox.ru 
реализации достаточно эффективным может оказаться метод производящего функционала, приведенный в работе [2], в которой показано, что для расчета термодинамического потенциала системы с взаимодействием заданного вида необходимо найти производящий функционал неприводимых частей оператора Мацубары. В указанной работе метод производящего функционала был изложен применительно к однокомпонентной системе, в которой сохраняется число частиц. В настоящей работе на примере хорошо изученной модели Фрелиха этот функционал рассчитан для двухкомпонентной системы с переменным числом частиц (фононов).

\section{2. МЕТОД ПРОИЗВОДЯШЕГО ФУНКЦИОНАЛА}

Для описания операторов и матриц, характеризующих систему многих частиц, мы используем метод производящего функционала, подробно рассмотренный в работе [2]. Приведем результаты и обозначения этой работы, необходимые в дальнейшем.

Операторы рождения и поглощения частиц в одночастичных состояниях, характеризуемых набором дискретных или непрерывных квантовых чисел $i$, обозначаются, как обычно, через $a^{+}(i)$ и $a(i)$ и подчиняются стандартным перестановочным соотношениям - коммутационным для бозонов и антикоммутационным для фермионов.

Производящий функционал матричных элементов произвольного оператора $A$ в представлении вторичного квантования имеет вид

$$
A\left(\xi^{*}, \lambda\right)=\left\langle 0\left|e^{\left(\xi^{*} a\right)} A e^{\left(a^{+} \lambda\right)}\right| 0\right\rangle,
$$

где $\xi^{*}(i)$ и $\lambda(i)$ - основные функции, зависящие от того же набора квантовых чисел, что и операторы рождения и поглощения, $(a b)$ - скалярное произведение $a$ и $b$ в гильбертовом пространстве одночастичных состояний. В случае статистики Ферми эти функции являются образующими грассмановой алгебры, антикоммутирующими между собой и с операторами рождения и поглощения ферми-частиц.

Обычно оператор $A$ может быть представлен в нормальной форме:

$$
A=\sum_{m, n}\left(\prod_{\{m\}} a^{+}(i) \tilde{A}(\{m\},\{n\}) \prod_{\{n\}} a(j)\right) .
$$

Величины $\tilde{A}(\{m\},\{n\})$ (функции системы квантовых чисел) являются коэффициентами нормального разложения. Иногда из этих коэффициентов выделяется множитель $(m ! n !)^{-1 / 2}$ или $(m ! n !)^{-1}$. Из соотношений

$$
e^{\left(\xi^{*} a\right)} a^{+}(i) e^{-\left(\xi^{*} a\right)}=a^{+}(i)+\xi^{*}(i), \quad e^{-\left(a^{+} \lambda\right)} a(i) e^{\left(a^{+} \lambda\right)}=a(i)+\lambda(i),
$$

которые можно установить с помощью разложения по функциям $\xi^{*}$ и $\lambda$, следует, что

$$
A\left(\xi^{*}, \lambda\right)=e^{\left(\xi^{*} \lambda\right)} \sum_{m, n}\left(\prod_{\{m\}} \xi^{*}(i) \tilde{A}(\{m\},\{n\}) \prod_{\{n\}} \lambda(j)\right) .
$$


Таким образом,

$$
\tilde{A}\left(\xi^{*}, \lambda\right)=e^{-\left(\xi^{*} \lambda\right)} A\left(\xi^{*}, \lambda\right)
$$

является производящим функционалом для коэффициентов нормального разложения. Обратное соотношение $A\left(\xi^{*}, \lambda\right)=e^{\left(\xi^{*} \lambda\right)} \tilde{A}\left(\xi^{*}, \lambda\right)$ также может оказаться полезным.

Вводя функциональную свертку

$$
A_{1}(\xi) \otimes A_{2}\left(\xi^{*}\right)=\left.A_{1}\left(\frac{\delta}{\delta \xi^{*}}\right) A_{2}\left(\xi^{*}\right)\right|_{\xi^{*}=0}=\left.A_{1}(\xi) A_{2}\left(\frac{\overleftarrow{\delta}}{\delta \xi}\right)\right|_{\xi=0}
$$

представим оператор $A$ в виде

$$
A=e^{\left(a^{+} \xi\right)} \otimes \tilde{A}\left(\xi^{*}, \lambda\right) \otimes e^{\left(\lambda^{*} a\right)},
$$

а след оператора - как

$$
\operatorname{Sp} A=A\left(\xi^{*}, \xi\right) \otimes,
$$

причем в случае статистики Ферми либо немая функция $\xi^{*}$ при свертке переносится циклически направо, либо функция $\xi$ - налево. Заметим, что немая функция всегда сворачивается со своей сопряженной. Из представления (6) следует соответствие

$$
A=A_{1} A_{2} \Longleftrightarrow A\left(\xi^{*}, \lambda\right)=A_{1}\left(\xi^{*}, \gamma\right) \otimes A_{2}\left(\gamma^{*}, \lambda\right) .
$$

Производящий функционал $B\left(\xi^{*}, \lambda\right)$ для неприводимых частей матричных элементов оператора $A$ определяется соотношением

$$
A\left(\xi^{*}, \lambda\right)=e^{B\left(\xi^{*}, \lambda\right)} .
$$

Если представить функционалы, входящие в выражения (5) и (6), через их неприводимые части, то свертка разобьется на произведение независимых (несвязанных) частей. Обычный подсчет множителей каждого типа приводит к теореме о связанных частях для следа оператора,

$$
\operatorname{Sp} A=e^{B\left(\xi^{*}, \xi\right)} \otimes=\exp \left(e^{B\left(\xi^{*}, \xi\right)} \otimes\right)_{\mathrm{c}},
$$

и для свертки двух функционалов,

$$
e^{B_{1}\left(\xi^{*}, \gamma\right)} \otimes e^{B_{2}\left(\gamma^{*}, \lambda\right)}=\exp \left(e^{B_{1}\left(\xi^{*}, \gamma\right)} \otimes e^{B_{2}\left(\gamma^{*}, \lambda\right)}\right)_{\mathrm{c}} .
$$

Функционал

$$
R\left(\xi^{*}, \lambda\right)=\operatorname{Sp} e^{\left(\xi^{*} a\right)} \rho e^{\left(a^{+} \lambda\right)}=\sum_{\{m\},\{n\}} \frac{\prod_{\{m\}} \xi^{*}(i) \prod_{\{n\}} \lambda(j)}{m ! n !} R_{m, n}(\{m\},\{n\}),
$$

где $\rho$ - оператор плотности, является производящим для частичных матриц плотности. Среднее значение оператора $A$ в произвольном смешанном состоянии при использовании оператора плотности определяется соотношением

$$
\langle A\rangle=\tilde{A}\left(\lambda^{*}, \xi\right) \otimes R\left(\xi^{*}, \lambda\right) .
$$


Вводя представление

$$
R\left(\xi^{*}, \lambda\right)=e^{\left(\xi^{*} \rho_{11} \lambda\right)+G\left(\xi^{*}, \lambda\right)},
$$

где $\rho_{11}$ - унарная матрица плотности, определим производящий функционал $G\left(\xi^{*}, \lambda\right)$ корреляционных матриц, являющихся неприводимыми частями многочастичных матриц плотности.

В случае системы, находящейся в термодинамически равновесном состоянии, термодинамический потенциал большого ансамбля имеет вид $\beta\left(\Omega-\Omega_{0}\right)=\left(e^{u\left(\nu^{*} \rho_{1}^{0}, \nu\right)} \otimes\right)_{c}$, производящий функционал всех корреляционных матриц равен

$$
\begin{aligned}
R & =e^{\left(\xi^{*} \rho_{11} \lambda\right)+G\left(\xi^{*}, \lambda\right)}=e^{\beta\left(\Omega-\Omega_{0}\right)+\left(\xi^{*} \rho_{1}^{0} \lambda\right)}\left(e^{u\left(\left(\xi^{*}+\nu^{*}\right) \rho_{1}^{0}, \nu+\left(1 \pm \rho_{1}^{0}\right) \lambda\right)} \otimes\right)_{\mathrm{c}}= \\
& =\exp \left(\left(\xi^{*} \rho_{1}^{0} \lambda\right)+\left(e^{u\left(\left(\xi^{*}+\nu^{*}\right) \rho_{1}^{0}, \nu+\left(1 \pm \rho_{1}^{0}\right) \lambda\right)} \otimes\right)_{\mathrm{c}}-\left(e^{u\left(\nu^{*} \rho_{1}^{0}, \nu\right)} \otimes\right)_{\mathrm{c}}\right)= \\
& =\exp \left(\left(\xi^{*} \rho_{1}^{0} \lambda\right)+\left(e^{u\left(\left(\xi^{*}+\nu^{*}\right) e^{-\beta(E-\mu)}\left(1 \pm \rho_{1}^{0}\right), \nu+\left(1 \pm \rho_{1}^{0}\right) \lambda\right)} \otimes\right)_{\mathrm{c}}-\left(e^{u\left(\nu^{*} \rho_{1}^{0}, \nu\right)} \otimes\right)_{\mathrm{c}}\right),
\end{aligned}
$$

где $u\left(\xi^{*}, \lambda\right)$ - производящий функционал неприводимых частей неэрмитова опе-

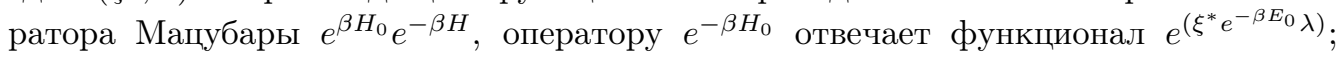
$\left(\xi^{*} \rho_{1}^{0} \lambda\right)=\left(\xi^{*} \frac{1}{e^{\beta T} \pm 1} \lambda\right), T=E-\mu$; знак в \pm определяется, как обычно, статистикой. Если отсчитывать энергию частиц от уровня химического потенциала $\mu$, то $T=E$. Это разложение всех матриц плотности является квантовым обобщением разложения частичных функций распределения по плотности и переходит в него в классическом пределе.

В работе [2] получено также уравнение для производящего функционала неприводимой части оператора Мацубары. Из уравнения Блоха для равновесного статистического оператора

$$
\frac{\partial \rho}{\partial \beta}=-H \rho, \quad H=T+V, \quad T=H_{0}-N \mu,
$$

следует уравнение

$$
\frac{\partial S}{\partial \beta}=-e^{\beta T} V e^{-\beta T} S
$$

для оператора Мацубары $S$. Разложение формального решения этого уравнения в виде упорядоченной по мнимому времени экспоненты приводит к стандартному ряду теории возмущений в операторной форме. Теория возмущений для производящего функционала неприводимых частей матричных элементов оператора Мацубары также следует из этого уравнения. Выражая входящие в него операторы через соответствующие производящие функционалы, получим уравнение

$$
\begin{aligned}
e^{\left(\xi^{*} \lambda\right)} \frac{\partial \widetilde{S}}{\partial \beta} & =-\widetilde{V}\left(\xi^{*} e^{\beta T} ; e^{-\beta T} \nu\right) e^{\left(\xi^{*} \nu\right)} \otimes e^{\left(\nu^{*} \lambda\right)} \widetilde{S}\left(\nu^{*} ; \lambda \mid \beta\right)= \\
& =-e^{\left(\xi^{*} \lambda\right)} \widetilde{V}\left(\xi^{*} e^{\beta T} ; e^{-\beta T}(\nu+\lambda)\right) \otimes \widetilde{S}\left(\nu^{*}+\xi^{*} ; \lambda \mid \beta\right)= \\
& =-\widetilde{V}\left(\xi^{*} e^{\beta T} ; e^{-\beta T}\left(\frac{\delta}{\delta \xi^{*}}\right)\right) e^{\left(\xi^{*} \lambda\right)} \widetilde{S}\left(\xi^{*} ; \lambda\right)= \\
& =-e^{\left(\xi^{*} \lambda\right)} \widetilde{V}\left(\xi^{*} e^{\beta T} ; e^{-\beta T}\left(\lambda+\frac{\delta}{\delta \xi^{*}}\right)\right) \widetilde{S}\left(\xi^{*} ; \lambda\right) .
\end{aligned}
$$


Используя представление $S\left(\xi^{*}, \lambda\right)=e^{\left(\xi^{*} \lambda\right)} \widetilde{S}\left(\xi^{*}, \lambda\right)=e^{\left(\xi^{*} \lambda\right)+u\left(\xi^{*}, \lambda\right)}$ функционала $S$ через производящий функционал $u$ неприводимых частей и применяя соотношение

$$
e^{-u} \frac{\delta}{\delta \xi^{*}} e^{u}=\frac{\delta u}{\delta \xi^{*}}+\frac{\delta}{\delta \xi^{*}},
$$

придем к дифференциальному уравнению

$$
\frac{\partial u}{\partial \beta}=-\widetilde{V}\left(\xi^{*} e^{\beta T}, e^{-\beta T}\left(\lambda+\frac{\delta u}{\delta \xi^{*}}+\frac{\delta}{\delta \xi^{*}}\right)\right)
$$

из которого с учетом начального условия $u(\beta=0) \equiv u\left(\xi^{*}, \lambda \mid 0\right)=0$ следует интегральное уравнение

$$
u\left(\xi^{*}, \lambda \mid \beta\right)=-\int_{0}^{\beta} \widetilde{V}\left(\xi^{*} e^{\tau T}, e^{-\tau T}\left(\lambda+\frac{\delta u\left(\xi^{*} ; \lambda \mid \tau\right)}{\delta \xi^{*}}+\frac{\delta}{\delta \xi^{*}}\right)\right) d \tau .
$$

Полагая в нулевом приближении $u=0$, путем последовательных итераций придем к высокотемпературному разложению неприводимых частей матричных элементов оператора Мацубары. Если возмущение имеет характер полинома по свободным полям, то правые части полученных уравнений являются полиномами той же степени по неприводимым частям. Действительно, при отсутствии других множителей справа от произведения двух типичных комбинаций, входящих в эти выражения, получим

$$
\left(\lambda+\frac{\delta u}{\delta \xi^{*}}+\frac{\delta}{\delta \xi^{*}}\right)_{i}\left(\lambda+\frac{\delta u}{\delta \xi^{*}}+\frac{\delta}{\delta \xi^{*}}\right)_{k}=\lambda_{i} \lambda_{k}+\lambda_{i} \frac{\delta u}{\delta \xi_{k}^{*}}+\frac{\delta u}{\delta \xi_{i}^{*}} \lambda_{k}+\frac{\delta u}{\delta \xi_{i}^{*}} \frac{\delta u}{\delta \xi_{k}^{*}}+\frac{\delta^{2} u}{\delta \xi_{i}^{*} \delta \xi_{k}^{*}} .
$$

Произведение большего числа таких сомножителей раскрывается аналогично. Очевидно, что уравнение (18) является в общем случае нелинейным.

\section{3. ТЕОРИЯ ВОЗМУЩЕНИЙ ДЛЯ МОДЕЛИ ФРЕЛИХА}

В модели Фрелиха рассматривается двухкомпонентная система электронов и фононов, их рассеивание определяется электрон-фононным взаимодействием [3]. Оператор электрон-фононного взаимодействия для этой модели является полиномиальным и имеет вид

$$
V=\frac{1}{L^{3 / 2}} \sum_{p, k} g(\omega(k)) a^{+}(p+k) a(p)\left(\alpha^{+}(-k)+\alpha(k)\right),
$$

где $L^{3}$ - объем системы, $a^{+}$и $a$ - операторы рождения и поглощения электронов, $\alpha^{+}$и $\alpha$-операторы рождения и поглощения фононов, $\omega(-k)=\omega(k)$ - частота, спин электрона при рассеянии из состояния $p$ в состояние $p+k$ не изменяется. Существенно, что этот оператор сохраняет импульс и число электронов, но не сохраняет число фононов. Соответственно, функционал $\widetilde{V}$ имеет вид

$$
\widetilde{V}=\frac{1}{L^{3 / 2}} \sum_{p, k} g(\omega(k)) \xi_{\mathrm{e}}^{*}(p+k) \lambda_{\mathrm{e}}(p)\left(\xi_{\mathrm{f}}^{*}(-k)+\lambda_{\mathrm{f}}(k)\right),
$$


где индекс "e" относится к электронным (грассмановым), а индекс "f" - к фононным переменным. Так как взаимодействие не меняет числа электронов, то $u=\sum_{n \geqslant 1} u_{n}$, где $u_{n}$ имеет одинаковый порядок $n$ по каждой из функций $\xi_{\mathrm{e}}^{*}$ и $\lambda_{\mathrm{e}}$. Поэтому уравнение (17) распадается на независимые уравнения для каждого $n$ :

$$
\begin{aligned}
e^{\left(\xi_{\mathrm{f}}^{*} \lambda_{\mathrm{f}}\right)} & \frac{\partial u_{1}\left(\xi^{*}, \lambda \mid \beta\right)}{\partial \beta}=-\frac{1}{L^{3 / 2}} \sum_{p, k} g(\omega(k))\left(e^{\beta \omega(k)} \xi_{\mathrm{f}}^{*}(-k)+e^{-\beta \omega(k)} \frac{\delta}{\delta \xi_{\mathrm{f}}^{*}(k)}\right) \times \\
& \times e^{\left(\xi_{\mathrm{f}}^{*} \lambda_{\mathrm{f}}\right)} e^{\beta(E(p+k)-E(p))} \xi_{\mathrm{e}}^{*}(p+k) \frac{\delta u_{1}\left(\xi^{*}, \lambda \mid \beta\right)}{\delta \xi_{\mathrm{e}}^{*}(p)}- \\
& -\frac{e^{\left(\xi_{\mathrm{f}}^{*} \lambda_{\mathrm{f}}\right)}}{L^{3 / 2}} \sum_{p, k} g(\omega(k))\left(e^{\beta \omega(k)} \xi_{\mathrm{f}}^{*}(-k)+e^{-\beta \omega(k)} \lambda_{\mathrm{f}}(k)\right) e^{\beta(E(p+k)-E(p))} \xi_{\mathrm{e}}^{*}(p+k) \lambda_{\mathrm{e}}(p), \\
e^{\left(\xi_{\mathrm{f}}^{*} \lambda_{\mathrm{f}}\right)} & \frac{\partial u_{n}\left(\xi^{*}, \lambda \mid \beta\right)}{\partial \beta}=-\frac{1}{L^{3 / 2}} \sum_{p, k} g(\omega(k))\left(e^{\beta \omega(k)} \xi_{\mathrm{f}}^{*}(-k)+e^{-\beta \omega(k)} \frac{\delta}{\delta \xi_{\mathrm{f}}^{*}(k)}\right) \times \\
& \times e^{\left(\xi_{\mathrm{f}}^{*} \lambda_{\mathrm{f}}\right)} e^{\beta(E(p+k)-E(p))} \xi_{\mathrm{e}}^{*}(p+k) \frac{\delta u_{n}\left(\xi^{*}, \lambda \mid \beta\right)}{\delta \xi_{\mathrm{e}}^{*}(p)}- \\
& -\frac{e^{\left(\xi_{\mathrm{f}}^{*} \lambda_{\mathrm{f}}\right)}}{L^{3 / 2}} \sum_{p, k} g(\omega(k)) \xi_{\mathrm{e}}^{*}(p+k)\left(\sum_{n_{1}+n_{2}=n} \frac{\delta u_{n_{1}}\left(\xi^{*}, \lambda \mid \beta\right)}{\delta \xi_{\mathrm{e}}^{*}(p)} \frac{\delta u_{n_{2}}\left(\xi^{*}, \lambda \mid \beta\right)}{\xi_{\mathrm{f}}^{*}(k)}+\right. \\
& \left.+\lambda_{\mathrm{e}}(p) \frac{\delta u_{n-1}}{\delta \xi_{f(k)}^{*}}\right) e^{\beta(E(p+k)-E(p)-\omega(k))}, \quad n \geqslant 2,
\end{aligned}
$$

при начальных условиях $u_{n}\left(\xi^{*}, \lambda \mid 0\right)=0$ для всех $n$. Уравнение для $u_{1}$ является линейным неоднородным. При последовательном решении уравнений легко заметить, что каждое из уравнений для $u_{n}, n \geqslant 2$, также является линейным неоднородным, так как функционалы меньших порядков определяются на предыдущих шагах.

Все уравнения (20) имеют вид

$$
\frac{\partial u}{\partial \beta}=e^{-\left(\xi_{\mathrm{f}}^{*} \lambda_{\mathrm{f}}\right)} \frac{\partial W}{\partial \beta} e^{\left(\xi_{\mathrm{f}}^{*} \lambda_{\mathrm{f}}\right)} u+u_{0}
$$

где

$$
\begin{gathered}
W(\beta)=-\frac{1}{L^{3 / 2}} \sum_{p, k} g(\omega(k))\left(\frac{e^{\beta \Delta E_{+}}}{\Delta E_{+}} \xi_{\mathrm{f}}^{*}(-k)+\frac{e^{\beta \Delta E_{-}}}{\Delta E_{-}} \frac{\delta}{\delta \xi_{\mathrm{f}}^{*}(k)}\right) \xi_{\mathrm{e}}^{*}(p+k) \frac{\delta}{\delta \xi_{\mathrm{e}}^{*}(p)}, \\
\Delta E_{ \pm}=E(p+k)-E(p) \pm \omega(k) .
\end{gathered}
$$

Вне зависимости от малости какого-либо параметра формальное решение этого уравнения с начальным условием $u(\beta=0) \equiv u\left(\xi^{*}, \lambda \mid 0\right)=0$ имеет вид

$$
u\left(\xi^{*}, \lambda \mid \beta\right)=e^{-\left(\xi_{\mathrm{f}}^{*} \lambda_{\mathrm{f}}\right)} \int_{0}^{\beta} e^{W(\beta)-W(\tau)} e^{\left(\xi_{\mathrm{f}}^{*} \lambda_{\mathrm{f}}\right)} u_{0}\left(\xi^{*}, \lambda \mid \tau\right) d \tau .
$$

Оператор $\xi_{\mathrm{e}}^{*}(p+k) \cdot \delta / \delta \xi_{\mathrm{e}}^{*}(p)$, содержащийся в полученном выражении, изменяет импульс электрона: $\xi_{\mathrm{e}}^{*}(p) \rightarrow \xi_{\mathrm{e}}^{*}(p+k)$; многократному применению данного оператора $\prod_{i}\left(\xi_{\mathrm{e}}^{*}\left(p+k_{i}\right) \cdot \delta / \delta \xi_{\mathrm{e}}^{*}(p)\right)$ отвечает следующее изменение импульса электрона: 
$\xi_{\mathrm{e}}^{*}(p) \rightarrow \xi_{\mathrm{e}}^{*}\left(p+\sum_{i} k_{i}\right)$, и это требует рассмотрения соответствующих промежуточных состояний.

Заметим, что при равенстве нулю любого из энергетических знаменателей $\Delta E_{+}$ или $\Delta E_{-}$разность $W(\beta)-W(\tau)$ остается конечной.

Оператору $e^{-\beta H}$ соответствует функционал

$$
\exp \left(\xi^{*} e^{-\beta E_{0}}, \nu\right) \otimes \exp \left(\left(\nu^{*}, \lambda\right)+u\left(\nu^{*}, \lambda\right)\right)=\exp \left(\left(\xi^{*} e^{-\beta E_{0}}, \lambda\right)+u\left(\xi^{*} e^{-\beta E_{0}}, \lambda\right)\right) .
$$

Отсюда следует, что функционал $u_{1}$ позволяет определить возмущенную энергию одночастичных состояний как функцию импульса, а также взаимодействие электрона с фононами, приводящее к рассеянию, в том числе многократному. Функционалы $u_{n}, n \geqslant 2$, описывают эффективное (возмущенное) взаимодействие, парное и многоэлектронное, а также многоэлектронное взаимодействие с фононами.

Можно указать порядок слагаемых функционала $u_{n}$ не только по электронным, но и по фононным переменным: $u=\sum u_{n \mid l, m}$. Каждый такой функционал определяет эффективную вершину поглощения $m$ фононов и испускания $l$ фононов группой $n$ электронов. Члены с $l=m=0$ определяют эффективное взаимодействие групп электронов в электрон-фононной системе и поправку к энергии электрона.

Хотя полученное решение определяет все слагаемые эффективного взаимодействия и процессы электрон-фононного взаимодействия, его расшифровка далеко не очевидна. Простейший подход - разложение экспоненты в степенной ряд - после громоздких выкладок приводит к ряду теории возмущений.

Более наглядной является итерационная процедура получения последовательных приближений, основанная на переходе от дифференциального к интегральному уравнению (18), которое для рассматриваемой модели Фрелиха принимает вид

$$
\begin{aligned}
u\left(\xi^{*}, \lambda \mid \beta\right)= & -\frac{1}{L^{3 / 2}} \int_{0}^{\beta} \sum_{p, k} g(\omega(k)) \xi_{\mathrm{e}}^{*}(p+k)\left(\lambda_{\mathrm{e}}(p)+\frac{\delta u\left(\xi^{*}, \lambda \mid \tau\right)}{\delta \xi_{\mathrm{e}}^{*}(p)}\right) \times \\
& \times\left(\xi_{\mathrm{f}}^{*}(-k) e^{\tau \omega(k)}+\left(\lambda_{\mathrm{f}}(k)+\frac{\delta u\left(\xi^{*}, \lambda \mid \tau\right)}{\delta \xi_{\mathrm{f}}^{*}(k)}\right) e^{-\tau \omega(k)}+\right. \\
& \left.+\frac{\delta^{2} u\left(\xi^{*}, \lambda \mid \tau\right)}{\delta \xi_{\mathrm{e}}^{*}(p) \delta \xi_{\mathrm{f}}^{*}(k)} e^{-\tau \omega(k)}\right) e^{\tau(E(p+k)-E(p))} d \tau .
\end{aligned}
$$

Итерационную схему решения этого уравнения можно построить, полагая взаимодействие, т. е. параметр связи $g(\omega(k))$, малым. Каждому члену этого разложения можно дать определенную интерпретацию.

Выделяя из уравнения (23) члены определенного порядка, как и для дифференциального уравнения, получим систему независимых линейных интегральных уравнений, приводящих к итеррационному методу определения каждого из функционалов $u_{n}$ :

$$
\begin{gathered}
u_{1}\left(\xi^{*}, \lambda \mid \beta\right)=-\frac{1}{L^{3 / 2}} \sum_{p, k} g(\omega(k)) \xi_{\mathrm{e}}^{*}(p+k) \int_{0}^{\beta} \frac{\delta u_{1}\left(\xi^{*}, \lambda \mid \tau\right)}{\delta \xi_{\mathrm{e}}^{*}(p)} \times \\
\times\left(\xi_{\mathrm{f}}^{*}(-k) e^{\tau \omega(k)}+\lambda_{\mathrm{f}}(k) e^{-\tau \omega(k)}\right) e^{\tau(E(p+k)-E(p))} d \tau-
\end{gathered}
$$




$$
\begin{aligned}
&-\frac{1}{L^{3 / 2}} \sum_{p, k} g(\omega(k)) \xi_{\mathrm{e}}^{*}(p+k) \int_{0}^{\beta} \frac{\delta^{2} u_{1}\left(\xi^{*}, \lambda \mid \tau\right)}{\delta \xi_{\mathrm{e}}^{*}(p) \delta \xi_{\mathrm{f}}^{*}(k)} e^{\tau(E(p+k)-E(p)-\omega(k))} d \tau- \\
&-\frac{1}{L^{3 / 2}} \sum_{p, k} g(\omega(k)) \xi_{\mathrm{e}}^{*}(p+k) \lambda_{\mathrm{e}}(p) \times \\
& \times \int_{0}^{\beta}\left(\xi_{\mathrm{f}}^{*}(-k) e^{\tau \omega(k)}+\lambda_{\mathrm{f}}(k) e^{-\tau \omega(k)}\right) e^{\tau(E(p+k)-E(p))} d \tau, \\
& u_{n}\left(\xi^{*}, \lambda \mid \beta\right)=-\frac{1}{L^{3 / 2}} \sum_{p, k} g(\omega(k)) \xi_{\mathrm{e}}^{*}(p+k) \int_{0}^{\beta} \frac{\delta u_{n}\left(\xi^{*}, \lambda \mid \tau\right)}{\delta \xi_{\mathrm{e}}^{*}(p)} \times \\
& \times\left(\xi_{\mathrm{f}}^{*}(-k) e^{\tau \omega(k)}+\lambda_{\mathrm{f}}(k) e^{-\tau \omega(k)}\right) e^{\tau(E(p+k)-E(p))} d \tau- \\
&-\frac{1}{L^{3 / 2}} \sum_{p, k} g(\omega(k)) \xi_{\mathrm{e}}^{*}(p+k) \int_{0}^{\beta} \frac{\delta^{2} u_{n}\left(\xi^{*}, \lambda \mid \tau\right)}{\delta \xi_{\mathrm{e}}^{*}(p) \delta \xi_{\mathrm{f}}^{*}(k)} e^{\tau(E(p+k)-E(p)-\omega(k))} d \tau- \\
&-\frac{1}{L^{3 / 2}} \sum_{p, k} g(\omega(k)) \xi_{\mathrm{e}}^{*}(p+k) \lambda_{\mathrm{e}}(p) \int_{0}^{\beta} \frac{\delta u_{n-1}}{\delta \xi_{\mathrm{f}}^{*}(k)} e^{\tau(E(p+k)-E(p)-\omega(k))} d \tau- \\
&-\frac{1}{L^{3 / 2}} \sum_{p, k} g(\omega(k)) \xi_{\mathrm{e}}^{*}(p+k) \int_{0}^{\beta} \sum_{n_{1}+n_{2}=n} \frac{\delta u_{n_{1}}\left(\xi^{*}, \lambda \mid \tau\right)}{\delta \xi_{\mathrm{e}}^{*}(p)} \times \\
& \times \frac{\delta u_{n_{2}}\left(\xi^{*}, \lambda \mid \tau\right)}{\xi_{\mathrm{f}}^{*}(k)} e^{\tau(E(p+k)-E(p)-\omega(k))} d \tau, \quad n \geqslant 2 . \\
&
\end{aligned}
$$

Очевидно, что система интегральных уравнений для функционалов $u_{n}, n \geqslant 1$, как и система дифференциальных уравнений, является замкнутой, линейной и неоднородной.

Все члены разложения функционала $u\left(\xi^{*} e^{-\beta E}, \lambda\right)$, полученного из итеррационного решения уравнений (23) и (24), можно интерпретировать в терминах диаграммной техники [1]. Все диаграммы содержат линии, направленные по оси мнимого времени от 0 до $\beta$. Линий, имеющих обратное направление, нет. Электронные линии непрерывны, идут от 0 (функции $\lambda_{\mathrm{e}}$ ) до $\beta$ (функции $\xi_{\mathrm{e}}^{*}$ ), изменяя импульс в каждой вершине, внутренних электронных линий функционал $u$ не содержит, фононные линии начинаются и заканчиваются в 0 (функции $\left.\lambda_{\mathrm{f}}\right)$ и в $\beta$ (функции $\left.\xi_{\mathrm{f}}^{*}\right)$ или в вершинах диаграммы при значениях мнимого времени $0<\theta_{m}<\beta$. В каждой из $n$ вершин диаграммы порядка $n$ сохраняется импульс, вершина вносит множитель $-L^{-3 / 2} g(\omega)$, где $\omega=\omega(k)$ - частота входящего или выходящего фонона. Каждый отрезок линии, расположенный на отрезке мнимого времени длины $\tau_{m}=\theta_{m+1}-\theta_{m}$ вносит множитель $e^{-\tau_{m} E_{m}}$, где $E_{m}$ - сумма энергий всех линий, расположенных на этом отрезке, при этом $\theta_{n+1}=\beta$. По значениям всех $n+1$ переменных $\tau_{m}$ проводится интегрирование при дополнительном условии $\sum \tau_{m}=\beta$. Метод вычисления таких интегралов приведен в приложении. Очевидно, что интеграл по конечному промежутку от непрерывной и ограниченной положительной функции не имеет ни нулей, ни полюсов при конечном значении $\beta$. При суммировании по импульсам внутренних фононных линий $\sum_{k}(\ldots)$ переходит в $L^{3} /(2 \pi)^{3} \int(\ldots) d^{3} k$, где область интегрирования - зона Бриллюэна, или элементарная ячейка обратной решетки. 
При этом достаточно вычислить только связанные диаграммы, так как по теореме о связанных диаграммах $1+u\left(\xi^{*}, \lambda\right)=e^{u\left(\xi^{*}, \lambda\right)_{\text {c }}}$.

\section{4. ЭЛЕКТРОН-ФОНОННАЯ СИСТЕМА}

Решение уравнений (24) учитывает изменение состояния электрон-фононной системы, имеющей температуру $1 / \beta$, которое возникает в результате электрон-фононного взаимодействия. Поправки к выражению для частот фононов, не содержащие электронных линий, в разложении (24) отсутствуют.

Выражения для членов первого порядка получаются достаточно просто: они имеют вид

$$
\begin{gathered}
\sum \xi_{\mathrm{e}}^{*}(p) \xi_{\mathrm{f}}^{*}(k) e^{-\beta(E(p)+\omega)} \lambda_{\mathrm{e}}(p+k) g(\omega) \frac{e^{\beta\left(\Delta E^{\prime}+\omega\right)}-1}{\Delta E^{\prime}+\omega}, \\
\sum \xi_{\mathrm{e}}^{*}(p) e^{-\beta E(p)} \lambda_{\mathrm{e}}(p-k) \lambda_{\mathrm{f}}(k) g(\omega) \frac{e^{\beta\left(\Delta E^{\prime \prime}-\omega\right)}-1}{\Delta E^{\prime \prime}-\omega}, \\
\Delta E^{\prime}=E(p)-E(p+k), \quad \Delta E^{\prime \prime}=E(p)-E(p-k) .
\end{gathered}
$$

Для поправки, имеющей только одну электронную линию и не имеющей входящих и выходящих фононных линий, получим

$$
\sum_{p} \xi_{\mathrm{e}}^{*}(p) e^{-\beta E} \lambda_{\mathrm{e}}(p) \int \frac{g^{2}(\omega) d^{3} k}{(2 \pi)^{3}} F_{0}+\cdots
$$

где $F_{0}=\left(e^{\beta \widetilde{\Delta E}}-1-\beta \widetilde{\Delta E}\right) /(\widetilde{\Delta E})^{2}$ и $\widetilde{\Delta E}=E(p-k)+\omega(k)-E(p)$. Вместе с членом $\sum_{p} \xi_{\mathrm{e}}^{*}(p) e^{-\beta E} \lambda_{\mathrm{e}}(p)$, который возникает из выражения $e^{-\beta H_{0}}$, содержащего невозмущенный гамильтониан, это разложение определяет возмущенные одноэлектронные состояния $\widetilde{E}(p)$ в электрон-фононной системе:

$$
\sum_{p} \xi_{\mathrm{e}}^{*}(p) e^{-\beta \widetilde{E}} \lambda_{\mathrm{e}}(p)=\sum_{p} \xi_{\mathrm{e}}^{*}(p) e^{-\beta E}\left[1+\int \frac{g^{2}(\omega) d^{3} k}{(2 \pi)^{3}} F_{0}+\cdots\right] \lambda_{\mathrm{e}}(p) .
$$

Члены четвертого и более высоких порядков по $g(\omega)$ весьма громоздки, хотя метод их получения достаточно очевиден. Члены, имеющие одну электронную и одну входящую или выходящую фононную линию, описывают поправки к электрон-фононному гамильтониану взаимодействия, они имеют третий и более высокие порядки по $g(\omega)$. Члены, описывающие взаимодействие электрона с двумя и более фононами, имеют второй и более высокие порядки.

Во втором порядке процесс рассеяния фонона электроном описывается, как в любом варианте теории возмущений, двумя членами: переход $\left(p_{1}, k_{1}\right) \rightarrow\left(p_{2}, k_{2}\right)$, $p_{1}+k_{1}=p_{2}+k_{2}$ происходит либо путем поглощения первичного фонона с последующим испусканием рассеянного фонона через промежуточное состояние, в котором имеется электрон с импульсом $p^{\prime}=p_{1}+k_{1}=p_{2}+k_{2}$, либо путем испускания рассеянного фонона с последующим поглощением первичного фонона через промежуточное состояние, в котором имеются оба фонона и электрон с импульсом 
$p^{\prime \prime}=p_{1}-k_{2}=p_{2}-k_{1}$. Существенно, что $p^{\prime \prime} \rightarrow p^{\prime}$ при замене $k_{1} \rightarrow-k_{2}$. Эти члены имеют вид

$$
\sum \xi_{\mathrm{e}}^{*}\left(p_{2}\right) \lambda_{\mathrm{e}}\left(p_{1}\right) \xi_{\mathrm{f}}^{*}\left(k_{2}\right) \lambda_{\mathrm{f}}\left(k_{1}\right) \frac{g\left(\omega_{1}\right) g\left(\omega_{2}\right)}{L^{3}} e^{-\beta\left(E\left(p_{2}\right)+\omega_{2}\right)} F_{i}, \quad i=1,2,
$$

где

$$
\begin{aligned}
F_{1}= & \frac{e^{\beta(\Delta E+\Delta \omega)}}{(\Delta E+\Delta \omega)\left(E\left(p^{\prime}\right)-E\left(p_{1}\right)-\omega_{1}\right)}-\frac{1}{(\Delta E+\Delta \omega)\left(E\left(p^{\prime}\right)-E\left(p_{2}\right)-\omega_{2}\right)}+ \\
& +\frac{e^{-\beta\left(E\left(p^{\prime}\right)-E\left(p_{2}\right)-\omega_{2}\right)}}{\left(E\left(p^{\prime}\right)-E\left(p_{1}\right)-\omega_{1}\right)\left(E\left(p^{\prime}\right)-E\left(p_{2}\right)-\omega_{2}\right)}, \\
F_{2}= & \frac{e^{\beta(\Delta E+\Delta \omega)}}{(\Delta E+\Delta \omega)\left(E\left(p^{\prime \prime}\right)-E\left(p_{1}\right)+\omega_{2}\right)}-\frac{1}{(\Delta E+\Delta \omega)\left(E\left(p^{\prime \prime}\right)-E\left(p_{2}\right)+\omega_{1}\right)}+ \\
& +\frac{e^{-\beta\left(E\left(p^{\prime \prime}\right)-E\left(p_{2}\right)+\omega_{1}\right)}}{\left(E\left(p^{\prime \prime}\right)-E\left(p_{1}\right)+\omega_{2}\right)\left(E\left(p^{\prime \prime}\right)-E\left(p_{2}\right)+\omega_{1}\right)}, \\
& \Delta E=E\left(p_{2}\right)-E\left(p_{1}\right), \quad \Delta \omega=\omega_{2}-\omega_{1}=\omega\left(k_{2}\right)-\omega\left(k_{1}\right) .
\end{aligned}
$$

В пределе $\Delta E \rightarrow 0, \Delta \omega \rightarrow 0$, существенном при усреднении по фононным состояниям,

$$
\begin{aligned}
& F_{1} \rightarrow \frac{e^{\beta\left(E(p)+\omega-E\left(p^{\prime}\right)\right)}-1-\beta\left(E(p)+\omega-E\left(p^{\prime}\right)\right)}{\left(E(p)+\omega-E\left(p^{\prime}\right)\right)^{2}}, \\
& F_{2} \rightarrow \frac{e^{\beta\left(E(p)-\omega-E\left(p^{\prime \prime}\right)\right)}-1-\beta\left(E(p)-\omega-E\left(p^{\prime \prime}\right)\right)}{\left(E(p)-\omega-E\left(p^{\prime \prime}\right)\right)^{2}} .
\end{aligned}
$$

Два члена, описывающие испускание или поглощение двух фононов, получаются аналогичным образом.

Члены, содержащие две электронные линии и не содержащие внешних фононных линий, определяют электрон-электронное взаимодействие, возникающее при обмене фононами. Во втором порядке по $g(\omega)$ это взаимодействие определяется выражением

$$
\sum \xi_{\mathrm{e}}^{*}\left(p_{1}\right) \xi_{\mathrm{e}}^{*}\left(p_{2}\right) e^{-\beta\left(E\left(p_{1}\right)+E\left(p_{2}\right)\right)} \int \frac{g^{2}(\omega) d^{3} k}{(2 \pi)^{3}} \lambda_{\mathrm{e}}\left(p_{1}+k\right) \lambda_{\mathrm{e}}\left(p_{2}-k\right) F(p),
$$

где

$$
\begin{gathered}
F(p) \equiv F\left(p_{1}, p_{2}, k\right)=\frac{e^{\beta \Delta E}}{\Delta E\left(\Delta E_{2}+\omega\right)}+\frac{1}{\Delta E\left(\Delta E_{1}-\omega\right)}-\frac{e^{\beta \Delta E_{1}-\omega}}{\left(\Delta E_{1}-\omega\right)\left(\Delta E_{2}+\omega\right)}, \\
\Delta E=\Delta E_{1}+\Delta E_{2}, \quad \Delta E_{1}=E\left(p_{1}\right)-E\left(p_{1}+k\right), \quad \Delta E_{2}=E\left(p_{2}\right)-E\left(p_{2}-k\right) .
\end{gathered}
$$

Очевидно, что в этом выражении можно либо произвести переобозначение импульсов $p_{1} \leftrightarrow p_{2}$, либо симметризовать это выражение. При малых $\beta$ (высоких температурах) $F \approx \beta^{2} / 2+\left(2 \Delta E_{1}+\Delta E_{2}-\omega\right) \beta^{3} / 3 !+\cdots$, откуда видно, что при высоких температурах электрон-фононное взаимодействие слабо влияет на энергетический спектр системы, следовательно, справедливо квазиклассическое приближение.

Члены высших порядков описывают взаимодействие с обменом двумя и более фононами и взаимодействие, сопровождающееся или излучением, или поглощением, или рассеянием фононов. 


\section{5. ЭФФЕКТИВНОЕ ВЗАИМОДЕЙСТВИЕ ЭЛЕКТРОНОВ}

Усреднение распределения Гиббса электрон-фононной системы по состояниям фононов приводит к распределению системы электронов с эффективным взаимодействием. При использовании метода производящего функционала это усреднение сводится к вычислению связных сверток: $e^{u_{\mathrm{e}}\left(\xi_{\mathrm{e}}^{*} \rho_{1, \mathrm{e}}^{0}, \lambda_{\mathrm{e}}\right)}=\exp \left(e^{u_{\mathrm{e}}\left(\xi_{\mathrm{e}}^{*} \rho_{1, \mathrm{e}}^{0}, \lambda_{\mathrm{e}} ; \nu_{\mathrm{f}}^{*} \rho_{1, \mathrm{f}}^{0}, \nu_{\mathrm{f}}\right)} \otimes_{\nu}\right)_{\mathrm{c}}$, откуда

$$
u_{\mathrm{e}}\left(\xi_{\mathrm{e}}^{*} \rho_{1, \mathrm{e}}^{0}, \lambda_{\mathrm{e}}\right)=\left(e^{u_{\mathrm{e}}\left(\xi_{\mathrm{e}}^{*} \rho_{1, \mathrm{e}}^{0}, \lambda_{\mathrm{e}} ; \nu_{\mathrm{f}}^{*} \rho_{1, \mathrm{f}}^{0}, \nu_{\mathrm{f}}\right)} \underset{\nu}{\otimes}\right)_{\mathrm{c}}
$$

При этом $\nu_{\mathrm{f}}^{*}\left(k_{1}\right) \rho_{1, \mathrm{f}}^{0}\left(k_{1}\right) \otimes \nu_{\mathrm{f}}\left(k_{2}\right)=\delta\left(k_{1}-k_{2}\right) \rho_{1, \mathrm{f}}^{0}\left(k_{1}\right)$, и в подынтегральном выражении появляются дополнительные множители $\rho_{1, \mathrm{f}}^{0}\left(k_{i}\right)$ при свертке (замыкании) каждой пары из входящих и выходящих фононных линий (и исключении сворачиваемых функций) - на диаграммном языке эта линия модифицируется. Индексы "e" и "f" в дальнейшем можно опускать.

Выражения (25), описывающие рассеяние фонона электроном, при этой процедуре дают дополнительные поправки

$$
\left.\sum_{p} \xi^{*}(p) \lambda(p) \int \frac{g^{2}(\omega) d^{3} k}{(2 \pi)^{3}\left(e^{\beta \omega}-1\right)} F_{i}(p)\right|_{p=p^{\prime}=p^{\prime \prime}}
$$

к одноэлектронным энергиям (при использовании предельных значений функций $F_{i}$ вследствие четности частоты как функции импульса фонона можно положить $\left.p^{\prime}=p^{\prime \prime}\right)$. Окончательно эффективная одноэлектронная энергия $\bar{E}$ в электрон-электронной системе определится соотношением

$$
\begin{aligned}
\sum_{p} \xi_{\mathrm{e}}^{*}(p) e^{-\beta \bar{E}} \lambda_{\mathrm{e}}(p)= \\
\quad=\sum_{p} \xi_{\mathrm{e}}^{*}(p) e^{-\beta E}\left[1+\int \frac{g^{2}(\omega) d^{3} k}{(2 \pi)^{3}}\left(F_{0}+\frac{F_{1}+F_{2}}{e^{\beta \omega}-1}\right) \ldots\right] \lambda_{\mathrm{e}}(p) .
\end{aligned}
$$

Усреднение по фононным состояниям добавит дополнительные члены (второго и более высокого порядков) в выражение (26) для эффективного взаимодействия за счет свертки членов, описывающих испускание и поглощение одного фонона:

$$
\begin{gathered}
\sum_{p} \xi_{\mathrm{e}}^{*}\left(p_{1}\right) \xi_{\mathrm{e}}^{*}\left(p_{2}\right) e^{-\beta\left(E\left(p_{1}\right)+E\left(p_{2}\right)\right)} \int \frac{g^{2}(\omega) d^{3} k}{(2 \pi)^{3}\left(e^{\beta \omega}-1\right)} \lambda_{\mathrm{e}}\left(p_{1}+k\right) \lambda_{\mathrm{e}}\left(p_{2}-k\right) \times \\
\times \frac{\left(e^{\beta\left(\Delta E_{1}+\omega\right)}-1\right)\left(e^{\beta\left(\Delta E_{2}-\omega\right)}-1\right)}{\left(\Delta E_{1}+\omega\right)\left(\Delta E_{2}-\omega\right)}+\cdots
\end{gathered}
$$

Эффективное взаимодействие в электронной системе (после усреднения по состояниям фононов) задается суммой выражений (23) и (26). 


\section{6. ЗАКЛЮЧЕНИЕ}

Bсе члены ряда теории возмущений для оператора Мацубары существенно зависят от температуры. Это связано с тем обстоятельством, что полученное разложение определяет не чистые возмущенные состояния, а возмущенную матрицу плотности системы, имеющей определенную температуру. Это разложение позволяет вычислять термодинамические функции электронного газа на основе данных о структуре кристалла, используя приведенный в работе [2] метод разложения термодинамического потенциала по неприводимым частям производящего функционала оператора Мацубары.

В приложениях могут быть использованы и модель электронн-фононной системы с явным учетом фононных состояний, и модель электронной системы с эффективным взаимодействием, появляющимся при усреднении по фононным состояниям. В случае электрон-фононной системы возмущение приводит к эффективному взаимодействию электронов при сохранении спектра фононов. В случае усреднения по состояниям фононов возникает дополнительная энергия электронов и энергия их взаимодействия. Ясно, что при этом изменится и эффективная одночастичная энергия электронов, и эффективный потенциал их взаимодействия. Выше приведены поправки второго порядка по параметру электрон-фононной связи как для одного, так и для второго случая. Члены более высокого порядка весьма громоздки, поэтому мы не выписали их в явном виде, хотя их вычисление может быть произведено теми же методами.

Наконец, следует иметь в виду, что всюду в приводимых расчетах определялись члены ряда для функционала $u\left(\xi^{*} e^{-\beta E}, \lambda\right)$, выражение для функционала $u\left(\xi^{*}, \lambda\right)$ может быть получено отсюда элементарно.

\section{ПРИЛОЖКНИЕ}

Интегралы вида $\int \exp \left(-\sum_{k} a_{k} \tau_{k}\right) \prod_{k} d \tau_{k}$ при условии $\sum_{k} \tau_{k}=\beta$ в общем случае при всех $a_{k}>0$ преобразуются следующим образом:

$$
\begin{aligned}
& \int \delta\left(\sum_{k} \tau_{k}-\beta\right) \exp \left(-\sum_{k} a_{k} \tau_{k}\right) \prod_{k} d \tau_{k}= \\
& \quad=\prod_{k} \int_{0}^{\infty} e^{-a_{k} \tau_{k}} d \tau_{k} \int \exp \left(i z\left(\beta-\sum_{k} \tau_{k}\right)\right) \frac{d z}{2 \pi}= \\
& \quad=\int e^{i z \beta} \frac{d z}{2 \pi} \prod_{k} \frac{1}{i z+a_{k}}=\sum_{k} \frac{e^{-a_{k} \beta}}{\prod_{m \neq k}\left(a_{m}-a_{k}\right)}
\end{aligned}
$$

(контур интегрирования по $z$ замыкается в верхней полуплоскости).

При совпадении некоторых величин $a_{k}$, например $a_{1}$ и $a_{2}$, получим

$$
\frac{F\left(a_{1}\right)}{a_{2}-a_{1}}+\frac{F\left(a_{2}\right)}{a_{1}-a_{2}} \rightarrow-\frac{\partial F\left(a_{1}\right)}{\partial a_{1}}=\frac{e^{-a_{1} \beta}}{\prod_{m>2}\left(a_{m}-a_{1}\right)}\left(\beta-\sum_{m>2} \frac{1}{a_{m}-a_{1}}\right)
$$

либо непосредственно, либо вычислением вычета в полюсе второго порядка. При совпадении большего числа полюсов выкладки аналогичны. Если выполнены не все 
условия $a_{k}>0$, то эти выражения являются аналитическим продолжением полученных значений интегралов.

\section{Список литературы}

[1] Л. Д. Ландау, Е. М. Лифшиц, Л. П. Питаевский, Теоретическая физика Т. 9. Статистическая физика. Ч. 2. Теория конденсированного состояния, Наука, М., 1978.

[2] Э. А. Аринштейн, ТМФ, 130:1 (2002), 54-63.

[3] Дж. Займан, Приниипы теории твердого тела, Мир, М., 1974.

Поступила в редакцию 10.07.2008, после доработки 5.05.2009 